\title{
Complementarity between medical geneticists and genetic counsellors: its added value in genetic services in Europe
}

\author{
Milena Paneque ${ }^{\star, 1,2,3}$, Clara Serra-Juhée, ${ }^{4,5}$, Rebecka Pestoff ${ }^{6,7}$, Christophe Cordier ${ }^{8}$, João Silva ${ }^{1,2,3}$, \\ Ramona Moldovan ${ }^{9}$ and Charlotta Ingvoldstad ${ }^{10,11,12}$
}

Clinical genetic services have progressed significantly the last few decades. This has led to the need for non-medical health-care professionals working as genetic counsellors in Europe and worldwide. However, there is no unified approach to genetic counsellors' role in health-care services in Europe, as in most countries the profession is still emerging and the educational backgrounds diverge noticeably, within and between countries. This qualitative study aims to describe the potential added value of genetic counsellors in clinical genetics teams and to explore their tasks and responsibilities in different European countries. A total of 143 participants providing genetic counselling in Europe at the time of the survey responded. The results show differences in activities of genetic counsellors, although there is a wide range of roles, which are similar. The ability to establish a quality relationship with consultands was frequently mentioned as one of the strengths of genetic counsellors, as well as a patient-centred approach. It is believed that genetic counsellors add a more holistic approach of psychosocial and familial dimensions of genetic concerns to the multidisciplinary teams. This study provides examples of successful integration of genetic counsellors in teams, as complementariness with medical geneticist became clear in several cases. Although the added value of genetic counsellors was manifested, professional recognition of genetic counsellors across Europe is still needed in order to support the quality of patients care and safety of practice.

European Journal of Human Genetics (2017) 25, 918-923; doi:10.1038/ejhg.2017.76; published online 17 May 2017

\section{INTRODUCTION}

Clinical genetic services and genetic counselling have developed significantly over the last few decades, particularly due to increased knowledge, discovery of new rare conditions, availability and complexity of new genetic tests, and new legislation. ${ }^{1-4}$ This has led to a surge of non-medical health-care professionals working as genetic counsellors in Europe and worldwide. ${ }^{5,6}$ Nevertheless, there is still no unified approach in Europe to genetic counsellors' role or educational backgrounds, and experience may vary considerably, within as well as between countries.

The European recommendations for genetic testing ${ }^{4}$ require that genetic testing is accompanied by genetic counselling and informed consent from the patient, and that professionals providing genetic counselling have specific training. A genetic test or a genetic condition may have an impact on many aspects of a person's life, such as psychological, medical, social and practical. ${ }^{8}$ It is the responsibility of the genetic counsellor to ensure the patient is appropriately equipped to manage potential implications, ${ }^{6,9,10}$ and adequate training, personal and professional competences are necessary to be able to do this. ${ }^{11,12}$ Development of such competences relies upon specific educational pathways, a defined professional role ${ }^{10}$ and a regulatory system for the profession (ie, the European registration system for genetic counsellors initiated in 2014). ${ }^{6}$ In most European countries the genetic counsellor profession is emerging, and the role, educational pathways and quality standards are still unclear. ${ }^{9,13-15}$

In an attempt to reflect the diverse context for genetic counselling in Europe this study includes perspectives from both medical doctors and genetic counsellors and from many European countries, aspects not consolidated in previous publications in the field. ${ }^{9,13}$

The main objectives of this study are to better understand the potential added value of genetic counsellors in the genetics healthcare services and to explore in depth their activities and tasks in different European countries. Specifically, we assess (1) clinical geneticists' and genetic counsellors' views on genetic counsellors' contribution during the sessions and team work and (2) the main differences between genetic counsellors' and medical geneticists' professional activities.

${ }^{1}$ i3S - Instituto de Investigação e Inovação em Saúde, Universidade do Porto, Porto, Portugal; ${ }^{2}$ IBMC - Institute for Molecular and Cell Biology, Universidade do Porto, Porto, Portugal; ${ }^{3}$ Centre for Predictive and Preventive Genetics (CGPP), Universidade do Porto, Porto, Portugal; ${ }^{4}$ Genetics Unit, Universitat Pompeu Fabra - Hospital del Mar Research Institute (IMIM), Barcelona, Spain; ${ }^{5}$ Centro de Investigación Biomédica en Red de Enfermedades Raras (CIBERER), Instituto de Salud Carlos III, Madrid, Spain; ${ }^{6}$ Department of Clinical Genetics, Linköping University Hospital, Linköping, Sweden; ${ }^{7}$ Department of Clinical and Experimental Medicine, Linköping University, Linköping, Sweden; ${ }^{8}$ Synlab Genetics, Department of Genetics, Lausanne, Switzerland; ${ }^{9}$ Department of Psychology, Babeş-Bolyai University, Cluj-Napoca, Romania; ${ }^{10}$ Department of Public Health and Caring Science, Uppsala University, Uppsala, Sweden; ${ }^{11}$ Department of Clinical Science, Intervention and Technology, Karolinska Institute, Stockholm, Sweden; ${ }^{12}$ Department of Women's and Children's Health, Uppsala University, Uppsala, Sweden

*Correspondence: Professor M Paneque, i3S - Instituto de Investigação e Inovação em Saúde, IBMC - Institute for Molecular and Cell Biology and Centre for Predictive and Preventive Genetics (CGPP), Universidade do Porto, Rua Júlio Amaral de Carvalho 45, Porto, 4200-135 Portugal; Phone: +351 226074942 ; Fax: +351 22 6099157; E-mail: milenaph@ibmc.up.pt

Received 19 December 2016; revised 21 February 2017; accepted 5 April 2017; published online 17 May 2017 


\section{METHODS}

\section{Participants}

Potential participants were ascertained via national and international associations, that is, National Associations of Human Genetics, the European network of genetic nurses and counsellors and the European Society of Human Genetics. Participants were eligible if they provided genetic counselling at the time of the survey in a European country.

\section{Measures}

This study was based on the qualitative part of a mixed-method study. Participants were asked to fill in a purposefully designed questionnaire aimed at assessing their role as a genetic counsellor and/or their experience in providing genetic counselling in their own country. The questions covered genetic counsellors' tasks and responsibilities. The questionnaire was developed based on a previous measure ${ }^{9}$ and included closed and open-ended questions.

\section{Procedure}

Professionals providing genetic counselling in European countries were recruited. After ethical approval was obtained (Ethical Review board of Plymouth University, reference No. 14/15-433, on 21 July 2015) participants received an invitation letter, a consent form and a link to the online survey via email. The sampling was based on a snowball technique. Everyone was sent a reminder letter after 4 weeks. Participation was anonymous and voluntary, but participants could opt to leave their contact details for further questions later. It was estimated to take $20 \mathrm{~min}$ to complete the questionnaire. Data collection was supported by the data collection tool in Google Drive (www.google.com).

\section{Data analysis}

Qualitative methods are particularly useful whenever there is little evidence about a given area or when an in depth exploration of a given topic is relevant. Therefore, we conducted a qualitative study to explore participant's perceptions on the added value of genetic counsellors to genetic health-care services in Europe.

Thematic analysis was conducted, by three experienced researchers (CS, JS and MP) who each analysed answers to one, of the three, open-ended questions, as described by Braun and Clarke. ${ }^{16}$ The method involves becoming familiar with the data, coding each statement and identifying codes that are relevant to the research question. The codes are grouped to find patterns of meaning that are organized under key themes (Table 2). Emerging themes are compared to develop content categories and, through a deductive approach, frameworks are constructed. The themes were cross-examined by all seven authors, then defined and given a descriptive name. ${ }^{16}$

\section{RESULTS}

A total of 143 participants responded to the online survey (Figure 1). Participant demographics are presented in Table 1.

Results are presented with descriptive quotes, each one followed by their professional code (GC for genetic counsellor; MG for medical

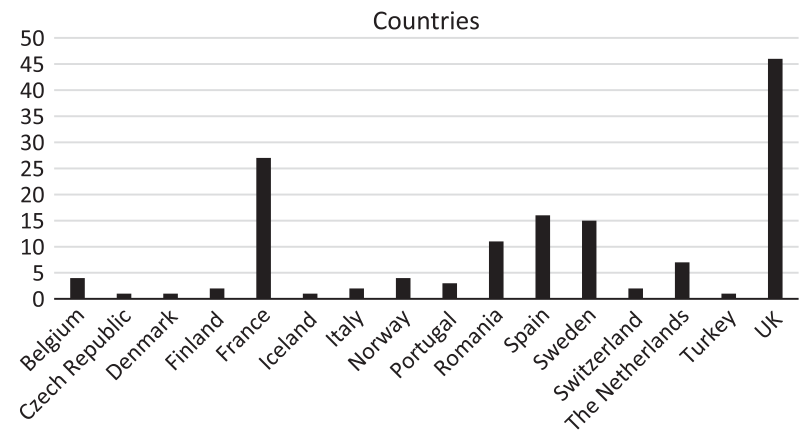

Figure 1 Countries of respondents. geneticist; and OP for other health-care professionals) and country. Qualitative assessment allowed the identification of major themes and sub-themes (Table 2).

What does a genetic counsellor adds to the clinical genetics team? Analysis of this question rendered three main themes: (1) remarkable contribution to the quality of the work done in the unit; (2) adding dimensions and perspectives not considered by other professionals; and (3) improvement in the accessibility of the services to patients and families.

The first emerging theme includes several aspects. Genetic counsellors, as well as medical geneticists, agreed genetic counsellors frequently acts as 'patient advocate', with a patient-centred approach, focussing on patients' needs and expectations in the session, as described by the following quotes:

'GCs are more in tune with the patients' needs and points of view. GCs act as patient advocates' GC, UK

\section{'Best understanding of patients, best advocate' GC, France}

Participants also highlighted the communication and counselling skills of genetic counsellors and the quality that counselling supervision entails for the practice; that expertise was linked to specific training:

\section{Table 1 Participants' sociodemographic data}

\begin{tabular}{lrc}
\hline Demographics & $\mathrm{n}$ & $\%$ \\
\hline Gender $(\mathrm{n}=143)$ & & \\
$\quad$ Male & 20 & 14 \\
Female & 122 & 85.3 \\
Unknown & 1 & 0.7 \\
& & \\
Age $(\mathrm{n}=143)$ & & 0 \\
$20-25$ & 0 & 11.9 \\
$26-30$ & 17 & 21 \\
$31-35$ & 30 & 19.6 \\
$36-40$ & 28 & 7.6 \\
$41-45$ & 11 & 11.2 \\
$46-50$ & 16 & 13.3 \\
$51-55$ & 19 & 8.4 \\
$56-60$ & 12 & 7 \\
Over 60 & 10 &
\end{tabular}

Professional qualifications $(\mathrm{n}=113$ )

Bachelor degree

Master degree

PhD/Doctorate

$\begin{array}{rr}3 & 2.7 \\ 78 & 69 \\ 32 & 28 .\end{array}$

Professional background $(\mathrm{n}=143)$

Medical geneticists

Genetic counsellors

Other professionals:

Psychologists

Nurses

Biologists

Years working $(n=142)$

$<1$ year

Between 2 and 5 years

Between 6 and 10 years

33.1

Over than 10 years

\section{2}

.
0.3 (1) .9

\section{6}


Table 2 Major themes and sub-themes that emerged from qualitative assessment

\begin{tabular}{|c|c|c|}
\hline Question & Major themes & Sub-themes \\
\hline \multirow[t]{3}{*}{$\begin{array}{l}\text { What do you consider a genetic counsellor adds to the } \\
\text { clinical genetics' TEAM? }\end{array}$} & $\begin{array}{l}\text { (1) Remarkably contribution to the quality of the } \\
\text { work done in the unit }\end{array}$ & $\begin{array}{l}\text { A patient-centred approach } \\
\text { Specific training including } \\
\text { (a) Communication skills } \\
\text { (b) Counselling skills } \\
\text { (c) Counselling supervision }\end{array}$ \\
\hline & $\begin{array}{l}\text { (2) Addition of dimensions and perspectives not } \\
\text { considered by other professionals }\end{array}$ & $\begin{array}{l}\text { Psychosocial dimension } \\
\text { Ethical dimension (also regarding research) } \\
\text { Legal framework }\end{array}$ \\
\hline & $\begin{array}{l}\text { (3) Improvement of patients and families' } \\
\text { accessibility to the services }\end{array}$ & $\begin{array}{l}\text { Practical dimension (more time, more phone } \\
\text { access) } \\
\text { Quality manner, closeness to the family }\end{array}$ \\
\hline \multirow[t]{2}{*}{$\begin{array}{l}\text { What do you consider is the added value of genetic } \\
\text { counsellors to the genetic counselling sessions? }\end{array}$} & $\begin{array}{l}\text { (1) A qualitative contribution to the counselling } \\
\text { process during the session }\end{array}$ & $\begin{array}{l}\text { A patient-centred model of practice } \\
\text { Understanding of the holistic impact of } \\
\text { genetic diagnoses } \\
\text { Bringing the family dimension into the } \\
\text { session } \\
\text { Relevant role in providing information and } \\
\text { clarifying patient's doubts } \\
\text { Highly relevant skills } \\
\text { Remarks to listening and being empathetic } \\
\text { Non-directiveness of practice and patients } \\
\text { advocacy }\end{array}$ \\
\hline & $\begin{array}{l}\text { (2) The pertinence of genetic counsellor's } \\
\text { professional action while preparing the session }\end{array}$ & $\begin{array}{l}\text { Gathering family history } \\
\text { Preparing consultations } \\
\text { Consultand's follow-up arrangements }\end{array}$ \\
\hline $\begin{array}{l}\text { What do you consider are the main differences between } \\
\text { the genetic counsellors' and medical geneticists' tasks } \\
\text { in a genetic counselling situation? }\end{array}$ & $\begin{array}{l}\text { (1) Type and complexity of the counselling } \\
\text { situation } \\
\text { (2) Level of training on counselling skills and } \\
\text { non-directiveness of } \\
\text { (3) The level of autonomy }\end{array}$ & \\
\hline
\end{tabular}

'Often MG do not access counselling supervision and they do not demonstrate such reflective practice as genetic counsellors do' GC, UK

'Represent an alternative to medical and technical approaches, bringing another way to hear, and speak with patients and families' MG, France

'A GC has skills in communication and different reactions and feeling coming up in the counselling sessions' GC, Finland

Furthermore, genetic counsellors were described as providing additional perspectives and dimensions to the team. Respondents frequently mentioned the ability of genetic counsellors to get close to patients and families as a whole, highlighting the psychosocial dimension of the process. Genetic counsellor contributions also involved the ethical dimensions of the practice, including research in this area, as well as their knowledge regarding the legal framework:

'(...) knowledge about law in the field and interpretation and management of difficult situations' MG, France

'Focus on psychosocial and ethical issues, knowledge of research in these areas' GC, UK
Some participants recognized that the genetic counsellors added efficiency to the genetic team. Their influence in genetic services seems to enhance the attention to patients and families at a lower cost, and to provide more accessibility, reduced waiting times, providing autonomous consultations and handling many administrative tasks before and after the appointments.

'GCs allow access to clinical genetics service (to appropriate patients) at a lower cost' GC, UK

'I think GCs have a personal contribution in the session and they make sure the genetic test decision is best for the patient' GC, France

The accessibility of genetic counsellors for patients and families was recurrently highlighted by respondents, not only in a practical dimension (more time and more phone accessibility) but also from a quality perspective (the closeness to the family). This accessibility places them as the liaison in the consultation and contact person of long-term support and follow-up of patients and families. In fact, they are seen as having a key role in preparing and supporting patients along the whole process:

'He is more 'accessible' and plays the role of intermediary between medical team and family' GC, France 
'Guides the patient through the process of genetic counselling, spends time face to face or by phone with the patient before and during genetic counselling and when offered, through the process of genetic testing. (...) GC is more easily available than the medical doctor and is also available for relatives and other concerned family members' GC, Sweden

'Being a contact-person over time (...) a longer-term relationship, someone the patient can reach to for questions over time' OP, Sweden

What is the added value of genetic counsellors to a genetic counselling session? Analysis of this question identified two main themes: (1) a qualitative contribution to the counselling process; and (2) the importance of genetic counsellors' activities while preparing the session. The first theme is supported by several illustrations of how the contribution of genetic counsellors can be relevant in a patientcentred model of practice, emphasizing understanding of the holistic impact of genetic diagnoses.

'When there is a diagnosis, a GC is the ideal person to see a patient... can provide detailed information and offer psychosocial support' GC, UK

'A different point of view, emphasis on the non-medical aspects of the (genetic) problem' MG, Czech Republic

'GCs are mainly focused on helping the patients through a process where they are learning to live with the genetic information... in general, GCs are focusing on being supportive through every aspect of the patient's needs' GC, Norway

Genetic counsellors were recognized as the ones bringing the family dimension to the session, as well as providing information and clarifying patients' doubts.

'GCs have a better insight into the dynamics within each family' MG, Sweden

'They also have more sensitivity in giving the information in a staged manner, with appropriate words and really make sure that the patient can comprehend it' GC, Spain

'Sometimes people can ask 'stupid questions' as we are not doctors... although none of the questions are stupid...' GC, UK

While one clinical geneticist perceived no added value by genetic counsellors to the session, other respondents referred to some of the skills that genetic counsellors use in the session as highly relevant, especially concerning listening and empathy.

'The GC expertise is in the counselling skills, decision making and adjustment to a diagnosis/risk as well for assisting in communicating information to other family members... this is invaluable in helping people to continue to live their lives' GC, UK

'GC establishes a 'high quality' relationship with the patient, different in terms of time and empathy' OP, Italy

Non-directiveness of practice and patient advocacy in counselling were strongly associated with genetic counsellors' practice. Moreover, the multidisciplinary aspect that genetic counsellors bring to the session was perceived as ensuring safe practice and a source of continuing support for families and patients.

'Improve the attention given to the patient to facilitate an informed decision by giving information and discussing all the options with them' GC, Spain

'I think we are more able to pick up if someone is unsure about testing and we are able to advocate for them if testing isn't in their best interest' GC, UK

'Multidisciplinarity helps the family. It also ensures that safe practice is at the forefront of the patient journey' GC, UK

The second theme described genetic counsellors as having a key role in preparing consultations, gathering family history and other practical contributions such as administrative tasks and doctors' follow-up arrangements. Many of the comments from medical geneticists were focused on these aspects.

'Once a diagnosis is made, GCs are essential in organizing testing, gathering family and medical information, organizing future contacts' MG, France

'Complement the MG information given to the patient and family' MG, Spain

Some contradictory perceptions emerged when analysing responses regarding genetic counsellors' contribution to the sessions. The genetic counsellor was described as a facilitator with relevant communication skills, by some respondents, whilst others considered genetic counsellors to mainly have an administrative role.

What are the main differences between genetic counsellors' and medical geneticists' tasks in a genetic counselling situation? Analysis of this last query rendered three main themes: (1) type and complexity of the counselling situation; (2) differences in counselling training and non-directiveness of practice; and (3) according to the level of autonomy.

The first theme related to diagnostic ability and exclusive skills of medical geneticists regarding medical examination, dysmorphological assessments and prescription:

'The main difference is that only the medically trained geneticist can do medical examination... GC is likely to give more time and focus to the psychosocial implications of the diagnosis' GC, UK

'In theory, a GC should perhaps be better at the psychological aspect of genetic counselling, while the clinical geneticist should be better at the medical aspects (uncommon histopathological types, medical implications of oncological therapy, etc.)' MG, Sweden

In relation to the previous idea, several respondents associated medical geneticists' roles mainly to medical activities, and genetic counsellors to a more comprehensive approach and response to the emotional life of the patients and their families:

'I think a GC will attempt a holistic approach to the counselling situation and a MG will favour a medical approach' GC, UK

'The GC has a more global involvement with the patient by taking into account the beliefs, emotional and psychosocial aspects as well as 
the family, supporting also healthy family members that do not necessarily require medical attention' GC, Spain

Sometimes this difference was linked to the lack of time of medical specialists although other participants mentioned skills GCs have due to the specific training.

'I think the main difference is that GCs have more training in communication skills, emotional and psychological support' GC, Romania

'MGs are under greater pressure to diagnose, to define... they often have limited time and are the only team members who can perform essential tasks such as patient examination. GCs are trained to respond to the emotional life of the patient putting diagnosis as a secondary aim' GC, UK

'GCs can spend more time to explain and maybe with simpler words when MGs explain briefly and are not sure if consultands understand' GC, France

Some participants described genetic counsellors as a professional that enhances patients' autonomy, that spends more time listening and exploring the issues involved in their decision-making process, whilst medical geneticists have a more paternalistic approach.

'MGs are able to make a diagnosis and give more precise medical information but patients can't always ask questions...' GC, France

'GCs listen, explore feelings and give more autonomy to patients during their session. MGs take a more paternalistic approach where they diagnose patients and tell them what is best for them' GC, UK

Another difference between both professionals related to their level of autonomy, describing genetic counsellors as less autonomous professionals that need to discuss their cases with the medical geneticist:

'GCs are involved in less difficult cases, more some sort of standardised counselling sessions' GC, Netherlands

'There are considerable overlaps but if the patient needs to be examined or if there is a complex inherited medical condition, a $M G$ is needed' MG, UK

'Only doctors can diagnose. They work more with complex conditions or where there are extensive ethical/potential legal issues such as an official patient complaint' GC, UK

One medical geneticist perceived no differences between the both roles. However, the idea of complementariness between both professions was recurrent in all three questions in our study:

'MG, if trained, can perform genetic counselling sessions, GCs cannot make diagnosis or medical examinations. GCs and MGs can be complementary' GC, Portugal

'Our roles are complementary, two different jobs for the same genetic counselling service' GC, Switzerland

\section{DISCUSSION}

Clinical genetic services are clearly established in Europe, although the organization can be extremely varied. ${ }^{13,15,17}$ Genetic services are often coordinated by medical geneticists or doctors trained in other related specialties, such as obstetrics or paediatrics. In some cases even trained laboratory scientists are providing genetic services to patients before genetic testing. The situation is perhaps even more ambiguous regarding professionals who provide genetic counselling, as they have different backgrounds such as nursing, midwifery, biology or psychology.

Genetic nurses and counsellors have traditionally provided support for patients and families with genetic concerns, assisting medical colleagues before, during and after the clinical appointment. ${ }^{18,19}$ The range of tasks and level of autonomy in which non-medical professionals work at genetics services has increased exponentially. $5,17-19$ Nowadays, standards of competence, including the knowledge, skills and attitudes necessary to enable a professional to function effectively and safely in the field of genetics, are established and current master's courses in Europe endorse the published guidelines. $6,7,10$

Even though there is a wide range of roles, it seems unanimous that genetic counsellors are delivering genetic counselling in many healthcare settings where an established familial diagnosis exists, and therefore diagnostic skills are not required. ${ }^{5,17}$ Our findings show the development of genetic counselling as a profession in Europe, in line with other studies recently published. . $^{6,9,13,19}$

The theoretical components of existing genetic counsellor's educational programmes are quite similar between countries, however the clinical components of the educational courses are less well addressed. ${ }^{20}$ This might explain the seemingly diverse roles and wide spectrum of autonomy of genetic counsellors, as reported in this study.

Our results show that genetic counsellors perform many administrative tasks as previously described in a recent Swedish study. The genetic counsellors' time was allocated to administration, such as sample handling, billing, arranging appointments and general administration, instead of direct patient work. ${ }^{9}$

In addition, the ability to establish a quality relationship with consultands was considered one of the main contributions of genetic counsellors to the genetic service, also found in previous research. ${ }^{21}$ In fact, both genetic counsellors and medical geneticists agree that genetic counsellors are more accessible professionals, closer to patients and families. Interestingly, it has been shown that consultands perceive the quality of genetic counselling process according to the kind of relationship established with the professional in charge. ${ }^{12,22}$

The patient-centred approach used by counsellors was recurrently identified as an added value, in comparison to a more traditional paternalistic approach used by some medical doctors. The principle of beneficence with respect for confidentiality and individual autonomy guides genetic counselling practice. ${ }^{23}$ Genetic counsellors are supposed to facilitate decision making with the aim to help the individual or the family understand the options for dealing with the risk of recurrence, while choosing the options that seems appropriate to them at that time. ${ }^{24}$ Besides, genetic counsellors should be highly aware of their own personal values to not impose advice on consultands. ${ }^{23}$

The ability and skills to practice using a non-directive approach not only might influence the consultand's experience but also ensure safe practice in accordance with the 'Code of professional practice for genetic counsellors in Europe'. ${ }^{25}$ In relation to that, our results indicated that genetic counsellors seem to have a key role in patient advocacy, as reported in previous studies. ${ }^{9,13}$ These studies showed added value of genetic counsellors in genetic teams as they provide 
insight into each case, and create an understanding for each patient's unique situation. ${ }^{9}$

Main findings from this study reinforced the added value of genetic counsellor to the clinical services, regarding case management, having a more holistic, ethical and psychological perspective, being able to offer continuous support and build a relationship with the patient, and being more accessible than medical geneticists. ${ }^{9}$ The study provided as well good examples of successful integration of genetic counsellors at European genetic services. Nevertheless, more knowledge is needed about genetic service delivery and the role of genetic counsellors in many European countries, identifying effective models of practice and organization of services. This needs to be discussed in light of increasing demands on genetic services and well-known constraints on the number of professionals available for addressing the patient's and family's needs.

In order to ensure patients have access to coordinated care, reconfiguration of professional roles and responsibilities, and a greater sharing of expertise is required. ${ }^{1}$ Recently, the European Society of Human Genetics established the European Board of Medical Genetics, which has three sections, one for genetic nurses and genetic counsellors, one for clinical laboratory geneticists and one for medical geneticists: they work together to improve standards of genetic healthcare services in Europe. ${ }^{6}$ This study may contribute to ascertain learning needs and educational pathways of both medical geneticists and genetic counsellors.

\section{Complementariness between medical geneticists and genetic counsellors}

While medical geneticists have had the main medical responsibility in genetic services, genetic counsellors are expected to enrich multidisciplinary teams with a more holistic approach of psychosocial and familial dimensions of genetic concerns. In the European countries represented in our study genetic counsellors commonly work in multidisciplinary teams, rather than independently, as previously reported. ${ }^{17}$ Our findings support the use of multidisciplinary teams, to continuously improve access, continuity and safety of patient care. ${ }^{17}$ We believe that the findings of this study reinforce complementariness as a relevant notion for genetic services organizations, more than pointing to the differences between medical geneticists and counsellors. Evidence shows that genetic counsellors undertake a significant workload associated with direct patient care, when utilized in specialist genetic settings. ${ }^{13,17}$ The teams seem to improve with the presence of genetic counsellors, increasing their availability towards the patient, assuring better care at a lower cost and providing more accessibility as they reduce the waiting time. Both GC and MG working in multidisciplinary teams assure the quality of patient's care acknowledging medical and non-medical issues inherent to a genetic condition and bringing a holistic approach to all related medical, psychosocial, ethical and legal issues.

This paper aimed to better understand the value of genetic counsellors in genetics health-care services, and to explore their activities and tasks in different European countries. The contribution that genetic counsellors provide to genetics health-care services can be summarized as the provision of better care by highly skilled professionals at lower cost, securing greater accessibility and proximity with the patients and families complementing the work with medical geneticists, relevant for the quality of health-care service. All the above can be used to argue the need of increased integration of genetic counsellors in countries where they are under-utilized ${ }^{15}$ and the promotion of professional recognition of genetic counsellors across Europe and worldwide.

\section{CONFLICT OF INTEREST}

The authors declare no conflict of interest.

\section{ACKNOWLEDGEMENTS}

We acknowledge Professor Heather Skirton for her relevant contribution in developing genetic counselling profession and encouragement for European research on the field. Financial support was rendered from the Department of Clinical Genetics, and Department of Clinical and Experimental Medicine, Linköping University, Linköping, Sweden for the contributions of RP to this paper.

1 Battista RN, Blancquaert I, Laberge A-M, van Schendel N, Leduc N: Genetics in health care: an overview of current and emerging models. Public Health Genomics 2012; 15: 34-45.

2 Hannig V, Cohen M, Pfotenhauer J, Williams M, Morgan T, Phillips J: Expansion of genetic services utilizing a general genetic counseling clinic. J Genet Couns 2014; 23: 64-71.

3 Disease R About rare diseases. UK. 2014. Available at: www.raredisease.org.uk (accessed 12 September 2014).

4 EuroGentest. Harmonizing genetic testing across Europe. Available at: http://www. eurogentest.org/index.php?id $=154$ (accessed 11 November 2016),

5 Skirton H, Barnes C, Curtis G, Walford-Moore J: The role and practice of the genetic nurse: report of the AGNC Working Party. J Med Genet 1997; 34: $141-147$

6 Paneque M, Moldovan R, Cordier C et al: Development of a registration system for genetic counsellors and nurses in health-care services in Europe. Eur J Hum Genet 2016; 24: 312-314.

7 Skirton H, Patch C, Voelckel M-A: Using a community of practice to develop standards of practice and education for genetic counsellors in Europe. J Community Genet 2010; 1: 169-173.

8 Decruyenaere M, Evers-Kiebooms G, Cloostermans T et al: Psychological distress in the 5-year period after predictive testing for Huntington's disease. Eur J Hum Genet 2003; 11: $30-38$.

9 Pestoff R, Ingvoldstad C, Skirton H: Genetic counsellors in Sweden: their role and added value in the clinical setting. Eur J Hum Genet 2016; 24: 350-355.

10 Skirton H, Lewis C, Kent A, Coviello DA: Genetic education and the challenge of genomic medicine: development of core competences to support preparation of health professionals in Europe. Eur J Hum Genet 2010; 18: 972-977.

11 National society of genetic counselors. About genetic counselors. Available at: http:// www.nsgc.org/p/cm/ld/fid = 175 (accessed 11 November 2016).

12 Guimarães L, Sequeiros J, Skirton H, Paneque M: What Counts as effective genetic counselling for presymptomatic testing in late-onset disorders? A study of the consultand's perspective. J Genet Couns 2013; 22: 437-447.

13 Cordier C, Taris N, De Pauw A, Sobol H, Philip N, Voelckel M-A: French Professionals in genetic counselor careers. J Genet Couns 2013; 22: 844-848.

14 Paneque M, Mendes Á, Saraiva J, Sequeiros J: Genetic counseling in Portugal: education, practice and a developing profession. J Genet Couns 2015; 24 548-552.

15 Skirton H, Cordier C, Ingvoldstad C, Taris N, Benjamin C: The role of the genetic counsellor: a systematic review of research evidence. Eur J Hum Genet 2015; 23: 452-458.

16 Braun V, Clarke V: Using thematic analysis in psychology. Qual Res Psychol 2006; 3.

17 Skirton H, Kerzin-Storrar L, Barnes C et al: Building the genetic counsellor profession in the United Kingdom: two decades of growth and development. J Genet Couns 2013; 22: 902-906.

18 Skirton H, Kerzin-Storrar L, Patch C et al: Genetic counsellors: a registration system to assure competence in practice in the United kingdom. Community Genet 2003; 6 : 182-183.

19 Paneque M, Moldovan R, Cordier C et al: Genetic counselling profession in Europe. eLS 2016; 1-6.

20 Ingvoldstad C, Seven M, Taris N, Cordier C, Paneque M, Skirton H: Components of genetic counsellor education: a systematic review of the peer-reviewed literature J Community Genet 2016; 7: 107-118.

21 McAllister M, Payne K, Macleod R, Nicholls S, Donnai D, Davies L: What process attributes of clinical genetics services could maximise patient benefits? Eur J Hum Genet 2008; 16: 1467-1476.

22 Skirton H: The client's perspective of genetic counseling - a grounded theory study. J Genet Couns 2001; 10: 311-329.

23 Edwards J, Greenberg J, Sahhar M: Global awakening in genetic counseling. Nat Preced. Available at: http://hdl.handle.net/10101/npre.2008.1574.1. 2008.

24 Fraser FC: Genetic counseling. Am J Hum Genet 1974; 26: 636-659.

25 European Board of Medical Genetics. Code of professional practice for genetic counsellors in Europe 2013, Available at: https://www.eshg.org/fileadmin/GCGN_Downloads/EBMGCodeofprofessionalpracticeforgeneticcounsellorsinEurope.pdf (accessed on 7 November 2016). 